\title{
Energy distribution in the dyadosphere of a Reissner-Nordström black hole in Møller's prescription
}

\author{
Elias C. Vagenas ${ }^{1}$ \\ Nuclear and Particle Physics Section \\ Physics Department \\ University of Athens \\ GR-15771, Athens, Greece
}

\begin{abstract}
The energy and momentum distributions in the dyadosphere of a Reissner-Nordström black hole are evaluated. The Møller's energy-momentum complex is employed for this computation. The spacetime under study is modified due to the effects of vacuum fluctuations in the dyadosphere. Therefore, the corrected Reissner-Nordström black hole metric takes into account the first contribution of the weak field limit of one-loop QED. Furthermore, a comparison and a consequent connection between our results that those already existing in the literature is provided. We hypothesize that when the energy distribution is of specific form there is a relation that connects the coefficients in the Einstein's prescription with those in the Møller's prescription.
\end{abstract}

\footnotetext{
${ }^{1}$ evagenas@phys.uoa.gr
} 


\section{Introduction}

Energy-momentum localization has been one of the most interesting but also thorny problems for the General Theory of Relativity. A plethora of different attempts to solve this problem have led to inconclusive results till now. Energy-momentum complexes introduced first by Einstein, were the foremost endeavor to solve this problem. After that a large number of different expressions for the energy-momentum complexes were proposed. A drawback of this attempt was that energy-momentum complexes had to be computed in quasi-Cartesian coordinates. Møller proposed a new expression for an energy-momentum complex which could be utilized to any coordinate system. However, the idea of the energy-momentum complex was severely criticized for a number of reasons. Considerable attempts to deal with this problematic issue are also the quasi-local and the superenergetic quantities ${ }^{2}$.

Virbhadra and collaborators enlivened anew the concept of energy-momentum complexes [2]. Since then, numerous works have been performed on evaluating the energy and momentum distributions of several gravitational backgrounds using the energy-momentum complexes [3]. In support of the importance of the concept of energy-momentum complexes, Chang, Nester and Chen [4] proved that every energy-momentum complex is associated with a Hamiltonian boundary term. Thus, the energy-momentum complexes are quasi-local and acceptable.

In this paper we evaluate the energy and momentum density distributions of the dyadosphere of the modified Reissner-Nordström black hole metric. The modifications to the aforesaid metric show up because of the the first contribution of the weak field limit of one-loop QED. The prescription that is used in the present analysis, is the one introduced by Møller. The reasons for presenting here the Møller's description are: (a) the argument that it is not restricted to quasi-Cartesian coordinates and (b) a work of Lessner [5] who argues that the Møller's energy-momentum complex is a powerful concept of energy and momentum in General Theory of Relativity.

The remainder of the paper is organized as follows. In Section 1 we consider the concept of energy-momentum complexes in the framework of General Theory of Relativity. In Section 2 the Møller's energy-momentum complex is presented. In Section 3 we introduce the concept of dyadosphere for the Reissner-Nordström black hole and present the modified Reissner-Nordström black hole metric. In Section 4 we provide all results that already exist in the literature. In Section 5 we utilize Møller's prescription and thus we calculate

\footnotetext{
${ }^{2}$ For a list of references concerning all the aforementioned issues see [1].
} 
the energy and momentum density distributions of the afore-mentioned spacetime. Furthermore, we compare order by order the coefficients that appear in the expression for the energy in the Møller's prescription with those that appear when other prescriptions are utilized. A relation that connects Møller's coefficients with those of other prescriptions is given. Finally, Section 6 is devoted to a brief summary of results and concluding remarks.

\section{Energy-Momentum Complexes}

The conservation laws of energy and momentum for an isolated (closed), i.e. no external force acting on the system, physical system in the Special Theory of Relativity are expressed by a set of differential equations. Defining $T_{\nu}^{\mu}$ as the symmetric energy-momentum tensor of matter and all non-gravitational fields the conservation laws are given by

$$
T_{\nu, \mu}^{\mu} \equiv \frac{\partial T_{\nu}^{\mu}}{\partial x^{\mu}}=0
$$

where

$$
\rho=T_{t}^{t} \quad j^{i}=T_{t}^{i} \quad p_{i}=-T_{i}^{t}
$$

are the energy density, the energy current density, the momentum density, respectively, and Greek indices run over the spacetime labels while Latin indices run over the spatial coordinate values.

Making the transition from the Special to General Theory of Relativity one adopts a simplicity principle which is called principle of minimal gravitational coupling. As a result of this, the conservation equation is now written as

$$
T_{\nu ; \mu}^{\mu} \equiv \frac{1}{\sqrt{-g}} \frac{\partial}{\partial x^{\mu}}\left(\sqrt{-g} T_{\nu}^{\mu}\right)-\Gamma_{\nu \lambda}^{\kappa} T_{\kappa}^{\lambda}=0
$$

where $g$ is the determinant of the metric tensor $g_{\mu \nu}(x)$. The conservation equation may also be written as

$$
\frac{\partial}{\partial x^{\mu}}\left(\sqrt{-g} T_{\nu}^{\mu}\right)=\xi_{\nu}
$$

where

$$
\xi_{\nu}=\sqrt{-g} \Gamma_{\nu \lambda}^{\kappa} T_{\kappa}^{\lambda}
$$

is a non-tensorial object. For $\nu=t$ this means that the matter energy is not a conserved quantity for the physical system ${ }^{3}$. From a physical point of view this lack of energy conservation can be understood as the possibility of transforming matter energy into

\footnotetext{
${ }^{3}$ It is possible to restore the conservation law by introducing a local inertial system for which at a specific spacetime point $\xi_{\nu}=0$ but this equality by no means holds in general.
} 
gravitational energy and vice versa. However, this remains a problem and it is widely believed that in order to be solved one has to take into account the gravitational energy. By a well-known procedure, the non-tensorial object $\xi_{\nu}$ can be written as

$$
\xi_{\nu}=-\frac{\partial}{\partial x^{\mu}}\left(\sqrt{-g} \vartheta_{\nu}^{\mu}\right)
$$

where $\vartheta_{\nu}^{\mu}$ are certain functions of the metric tensor and its first order derivatives. Therefore, the energy-momentum tensor of matter $T_{\nu}^{\mu}$ is replaced by the expression

$$
\theta_{\nu}^{\mu}=\sqrt{-g}\left(T_{\nu}^{\mu}+\vartheta_{\nu}^{\mu}\right)
$$

which is called energy-momentum complex since it is a combination of the tensor $T_{\nu}^{\mu}$ and a pseudotensor $\vartheta_{\nu}^{\mu}$ which describes the energy and momentum of the gravitational field. The energy-momentum complex satisfies a conservation law in the ordinary sense, i.e.

$$
\theta_{\nu, \mu}^{\mu}=0
$$

and it can be written as

$$
\theta_{\nu}^{\mu}=\chi_{\nu, \lambda}^{\mu \lambda}
$$

where $\chi_{\nu}^{\mu \lambda}$ are called superpotentials and are functions of the metric tensor and its first order derivatives.

It is obvious that the energy-momentum complex is not uniquely determined by the condition that is usual divergence is zero since it can always been added to the energymomentum complex a quantity with an identically vanishing divergence.

\section{Møller's Prescription}

The energy-momentum complex of Møller in a four-dimensional background is given as [6]

$$
\mathcal{J}_{\nu}^{\mu}=\frac{1}{8 \pi} \xi_{\nu, \lambda}^{\mu \lambda}
$$

where the Møller's superpotential $\xi_{\nu}^{\mu \lambda}$ is of the form

$$
\xi_{\nu}^{\mu \lambda}=\sqrt{-g}\left(\frac{\partial g_{\nu \sigma}}{\partial x^{\kappa}}-\frac{\partial g_{\nu \kappa}}{\partial x^{\sigma}}\right) g^{\mu \kappa} g^{\lambda \sigma}
$$

with the antisymmetric property

$$
\xi_{\nu}^{\mu \lambda}=-\xi_{\nu}^{\lambda \mu}
$$


It is easily seen that the Møller's energy-momentum complex satisfies the local conservation equation

$$
\frac{\partial \mathcal{J}_{\nu}^{\mu}}{\partial x^{\mu}}=0
$$

where $\mathcal{J}_{0}^{0}$ is the energy density and $\mathcal{J}_{i}^{0}$ are the momentum density components.

Thus, the energy and momentum in Møller's prescription for a four-dimensional background are given by

$$
P_{\mu}=\iiint \mathcal{J}_{\mu}^{0} d x^{1} d x^{2} d x^{3}
$$

and specifically the energy of the physical system in a four-dimensional background is

$$
E=\iiint \mathcal{J}_{0}^{0} d x^{1} d x^{2} d x^{3}
$$

It should be noted that the calculations are not anymore restricted to quasi-Cartesian coordinates but they can be utilized in any coordinate system.

\section{The Dyadosphere of the Reissner-Nordström black hole}

The Reissner-Nordström black hole is described by the line element

$$
d s^{2}=f(r) d t^{2}-\frac{d r^{2}}{f(r)}-r^{2}\left(d \theta^{2}+\sin \theta d \phi^{2}\right)
$$

where the metric element $f(r)$ is given as

$$
f(r)=1-\frac{2 M}{r}+\frac{Q^{2}}{r^{2}}
$$

and the quantities $M$ and $Q$ are the mass and the electric charge, respectively, of the black hole. The black hole horizon of the Reissner-Nordström black hole is located at ${ }^{4}$

$$
r_{+}=M \pm \sqrt{M^{2}-Q^{2}}
$$

The concept of dyadosphere was first introduced by Ruffini [7], and also Preparata, Ruffini, and Xue [8]. They claimed that outside the black hole horizon there is a region where the electromagnetic field strength, namely the only nonvanishing radial component $\overrightarrow{\mathcal{E}}=\frac{Q}{r^{2}} \hat{r}$, is larger than the well-known Heisenberg-Euler critical value for the electron-positron pair creation

$$
\mathcal{E}_{\text {crit }}=\frac{m_{e}^{2} c^{3}}{\hbar e}
$$

\footnotetext{
${ }^{4}$ We have set $G=1$ and $c=1$.
} 
where $m_{e}$ and $e$ stand for the mass and the electric charge, respectively, of an electron. This region is called dyadosphere and it extends from the black hole horizon, i.e. $r_{+}$, which is the inner radius of the dyadosphere to an outer radius, $r_{d s}$, given by ${ }^{5}$

$$
r_{d s}=\sqrt{\left(\frac{\hbar}{m_{e} c}\right)\left(\frac{G M}{c^{2}}\right)\left(\frac{m_{p l}}{m_{e}}\right)\left(\frac{e}{q_{p l}}\right)\left(\frac{Q}{\sqrt{G} M}\right)}
$$

where $m_{p l}\left(=\sqrt{\frac{\hbar c}{G}}\right)$ and $q_{p l}(=\sqrt{\hbar c})$ are respectively the Planck mass and charge. It was found that the dyadosphere exists only for black holes with mass from the upper limit for neutron stars at $3.2 M_{\odot}$ all the way up to a maximum mass of $6 \cdot 10^{5} M_{\odot}$. On the outer radius of the dyadosphere the electromagnetic field strength becomes

$$
\mathcal{E}_{\text {crit }}=\frac{Q}{r_{d s}^{2}} .
$$

It was shown that the electron-positron pair creation processes occur over the entire dyadosphere. Thus an energy extraction process takes place due to the vacuum fluctuations in this region. It was claimed that this energy source might lead to a natural explanation for the gamma ray bursts as well as for the ultra high energy cosmic rays.

In the presence of a strong electromagnetic field, as that in the dyadosphere, the velocity of light propagation depends on the vacuum polarization states [9]. Drummond and Hathrell showed that the effect of the vacuum polarization may lead to superluminal photon propagation [10]. Daniels and Shore who investigated the photon propagation around a charged black hole proved that the effect of the one-loop vacuum polarization on photon propagation in the Reissner-Nordström black hole background makes the superluminal photon propagation possible [11]. Therefore, De Lorenci, Figueiredo, Fliche, and Novello were led to investigate the corrections for the Reissner-Nordström black hole metric due to the first contribution of the weak field limit of the one-loop QED [12]. They found that the metric element (17) is written as

$$
f(r)=1-\frac{2 M}{r}+\frac{Q^{2}}{r^{2}}-\frac{\sigma Q^{4}}{5 r^{5}} .
$$

The last term in (22) expresses the contribution coming from the one-loop QED in the first order of approximation. This term is of the same order of magnitude as the classical Reissner-Nordström charge term, i.e. the second term in (22), as shown in [12].

It is evident that the Reissner-Nordström case arises from the aforementioned metric element for the limit case $\sigma=0$.

\footnotetext{
${ }^{5}$ Here we have reinstated the constants $G$ and $c$ in order to make clear the hybrid gravitational and quantum nature of this quantity.
} 


\section{What has been done till now}

In 2003, Xulu evaluated the energy distribution in the dyadosphere for the modified Reissner-Nordström black hole metric [13]. For this computation, the energy-momentum prescriptions of Einstein, Landau-Lifshitz, Papapetrou, and Weinberg were employed. All four prescriptions gave the same and acceptable energy distribution which is of the form

$$
E_{\text {Einstein }}=E_{L L}=E_{\text {Pap }}=E_{\text {Wein }}=M-\frac{Q^{2}}{2 r}+\frac{\sigma Q^{4}}{10 r^{5}} \text {. }
$$

It is clear that in the dyadosphere (where $r$ is small) the last term in (23) plays an important role.

By setting $\sigma=0$, one gets the energy distribution for the Reissner-Nordström black hole metric as evaluated by Virbhadra [14], i.e.

$$
E_{R-N}=M-\frac{Q^{2}}{2 r}
$$

using the energy-momentum prescriptions of Einstein and Landau-Lifshitz.

\section{Energy distribution in Møller's Prescription}

The aim of this section is to evaluate the energy and momentum distributions associated with the modified Reissner-Nordström black hole metric, i.e. (16) and (22), using the Møller's energy-momentum complex. We first have to evaluate the superpotentials in the context of Møller's prescription. There are eight nonzero superpotentials

$$
\begin{aligned}
& \xi_{1}^{12}=-\xi_{1}^{21}=2\left(M-\frac{Q^{2}}{r}+\frac{3 \sigma Q^{4}}{5 r^{5}}\right) \sin \theta \\
& \xi_{3}^{23}=-\xi_{3}^{32}=2\left(2 M-\frac{Q^{2}}{r}+\frac{\sigma Q^{4}}{5 r^{5}}-r\right) \sin \theta \\
& \xi_{4}^{24}=-\xi_{4}^{42}=2\left(2 M-\frac{Q^{2}}{r}+\frac{\sigma Q^{4}}{5 r^{5}}-r\right) \sin \theta \\
& \xi_{4}^{34}=-\xi_{4}^{43}=-2 \cos \theta .
\end{aligned}
$$

By substituting the Møller's superpotentials, as given by (25), into equation (10), one gets the energy density distribution

$$
\mathcal{J}_{0}^{0}=\frac{Q^{4}\left(r^{4}-3 \sigma Q^{2}\right)}{4 \pi r^{6}} \sin \theta
$$

while the momentum density distributions take the form

$$
\begin{aligned}
\mathcal{J}_{1}^{0} & =0 \\
\mathcal{J}_{2}^{0} & =0 \\
\mathcal{J}_{3}^{0} & =0 .
\end{aligned}
$$


Therefore, if we substitute equation (26) into equation (15), we get the energy distribution of the modified Reissner-Nordström black hole that is contained in a "sphere" of radius $r$

$$
E(r)=M-\frac{Q^{2}}{r}+\frac{3 \sigma Q^{4}}{5 r^{5}}
$$

which is also the energy (mass) of the gravitational field that a neutral particle experiences at a finite distance $r$. Thus, the energy given by equation (30) is in addition called effective gravitational mass $\left(E=M_{e f f}\right)$ of the spacetime under study.

Additionally, if we replace equations (27, 29) into equation (14) we get the momentum components which are given by

$$
P_{1}=P_{2}=P_{3}=0
$$

It is obvious that imposing $\sigma=0$ in expression (30) one gets the energy distribution for the Reissner-Nordström black hole metric as evaluated by Virbhadra [15], i.e.

$$
E_{R-N}=M-\frac{Q^{2}}{r}
$$

using the energy-momentum prescription of Møller.

A couple of comments are in order. Firstly, a neutral test particle experiences at a finite distance $r$ the gravitational field of the effective gravitational mass described by expression (30). Secondly, the energy-momentum complex of Møller as formulated here for the modified Reissner-Nordström black hole due to the first contribution of the weak field limit of one-loop QED satisfies the local conservation laws

$$
\frac{\partial \mathcal{J}_{\nu}^{\mu}}{\partial x^{\mu}}=0
$$

Thirdly, it is easily seen that asymptotically the effective gravitational mass of the modified Reissner-Nordström black hole takes the value of the Schwarzschild mass

$$
E(r \rightarrow \infty)=M
$$

Fourthly, comparing order by order the expressions (23) and (30) it is evident that the coefficients of the r-terms are not equal. This discrepancy was identified some years ago when expressions for the Reissner-Nordström black hole, i.e. (24) and (32) were compared (see [15]). Therefore, we hypothesize that there is a connection between the coefficients of the expression for the energy (when the energy-momentum complex of Einstein is employed) of the form

$$
E(r)=\sum_{n=0}^{+\infty} \alpha_{n}^{(\text {Einstein })} r^{-n}
$$


and those of the expression for the energy (when the energy-momentum of Møller is employed) of the form

$$
E(r)=\sum_{n=0}^{+\infty} \alpha_{n}^{(M \varnothing l l e r)} r^{-n}
$$

The relation that materializes this connection between the aforementioned coefficients is given by

$$
\alpha_{n}^{(\text {Einstein })}=\frac{1}{n+1} \alpha_{n}^{(\text {Møller })} .
$$

By comparing the result presented in this work, i.e. expression (30), with the corresponding one that already exists in the literature, i.e. expression (23), is easily seen that relation (37) holds true. In addition, the hypothesis is true for the expressions associated with the Reissner-Nordström black hole, i.e. (24) and (32). Furthermore, in support of this hypothesis we provide one more example that already exists in the literature. It was shown by Radinschi [16] that the energy distribution of a charged regular black hole when the energy-momentum complex of Einstein is employed, is given by

$$
E(r)=M-\frac{Q^{2}}{2 r}+\frac{Q^{6}}{24 M^{2} r^{3}}-\frac{Q^{10}}{240 M^{4} r^{5}}+O\left(\frac{1}{r^{6}}\right)
$$

while the corresponding energy distribution when the energy-momentum complex of Møller is employed, is given by

$$
E(r)=M-\frac{Q^{2}}{r}+\frac{Q^{6}}{6 M^{2} r^{3}}-\frac{Q^{10}}{40 M^{4} r^{5}}+O\left(\frac{1}{r^{6}}\right)
$$

\section{Conclusions}

In this work, we briefly presented the concept of the dyadosphere. This is a region outside of the event horizon of the Reissner-Nordström black hole. In this region the electromagnetic field strength is overcritical and thus electron-positron pair creation processes take place. Therefore, the effects of the vacuum fluctuations in the dyadosphere modify the metric that describes the Reissner-Nordström black hole. We explicitly calculate the energy and momentum density distributions associated with the modified ReissnerNordström black hole metric. The Møller's energy-momentum complex is employed for this computation. The energy distribution derived here can be regarded as the effective gravitational mass experienced by a neutral test particle placed in the spacetime under consideration. In addition, setting the parameter $\sigma$ equals to zero, we derive the energy distribution of the Reissner-Nordström black hole. Furthermore, a comparison and a 
consequent connection between our results that those already existing in the literature is provided. We hypothesize that when the energy distribution is of specific form (see expressions (35) and (36) ) there is a relation that connects the coefficients in the Einstein's prescription with those in the Møller's prescription. This hypothesis is supported by the expressions for the energy of the Reissner-Nordström black hole as well as for the energy of a charged regular black hole. However, further investigation and verification is needed. We hope to return to this issue in a future work.

\section{Acknowledgements}

Research for ECV is supported by EPEAEK II in the framework of the grant PYTHAGORAS II - University Research Groups Support (co-financed $75 \%$ by EU funds and $25 \%$ by National funds).

\section{References}

[1] E.C. Vagenas, Int. J. Mod. Phys. D 14, 573 (2005).

[2] K.S. Virbhadra, Phys. Rev. D 42, 1066 (1990); K.S. Virbhadra, Pramana 38, 31 (1992); N. Rosen and K.S. Virbhadra, Gen. Rel. Grav. 25, 429 (1993); K.S. Virbhadra and J.C. Parikh, Phys. Lett. B 317, 312 (1993); K.S. Virbhadra and J.C. Parikh, Phys. Lett. B 331, 302 (1994); Erratum-ibid B 340, 265 (1994); K.S. Virbhadra, Pramana 45, 215 (1995); A. Chamorro and K.S. Virbhadra, Pramana-J. Phys. 45, 181 (1995); J.M. Aguirregabiria, A.Chamorro and K.S. Virbhadra, Gen. Rel. Grav. 28, 1393 (1996); A. Chamorro and K.S. Virbhadra, Int.J. Mod. Phys. D 5, 251 (1997); K.S. Virbhadra, Phys. Rev. D 60, 104041 (1999).

[3] I.-C. Yang, C.-T. Yeh, R.-R. Hsu and C.-R. Lee, Int. J. Mod. Phys. D 6, 349 (1997); I. Radinschi, Acta Phys. Slov. 49, 789 (1999); I. Radinschi, Mod. Phys. Lett. A 15, 803 (2000); I. Radinschi, Acta Phys. Slov. 50, 609 (2000); I. Radinschi, FizikaB 9, 43 (2000); I. Radinschi, Nuovo Cim. B 115, 501 (2000); I. Radinschi, Mod. Phys. Lett. A 15, 2171 (2000); I. Radinschi, Chin. J. Phys. 39, 231 (2001); I.-C. Yang and I. Radinschi, Chin. J. Phys. 41, 326 (2003); I.-C. Yang and I. Radinschi, Mod. Phys. Lett. A 17, 1159 (2002); I. Radinschi, Chin. J. Phys. 39, 393 (2001); I. C. Yang and I. Radinschi, Chin. J. Phys. 42, 40 (2004); I. Radinschi and I. C. Yang, FizikaB 14, 3 (2005); I. Radinschi, Rom. J. Phys. 50, 57 (2005); 
I. Radinschi, "Energy distribution of a black hole solution in Heterotic String theory", to be published in Quantum Cosmology Research Trends. Horizons in World Physics. Volume 246 (edited by Albert Reimer, Nova Science, 2005), gr-qc/0302047; I.-C. Yang and I. Radinschi, "The energy for $(2+1)$-dimensional black hole solutions", gr-qc/0309130 I. Radinschi and I. C. Yang, "On the energy of stringy black holes", arXiv:gr-qc/0405118; I. Radinschi and T. Grammenos, "Energy distribution of the Einstein-Klein-Gordon system for a static spherically symmetric spacetime in (2+1)-dimensions", arXiv:gr-qc/0508005.; I.-C. Yang, Chin. J. Phys. 38, 1040 (2000); O. Aydogdu and M. Salti, "Energy imparted by gravitational waves in inhomogeneous space-times", arXiv:gr-qc/0602012; M. Salti, "Teleparallel Gravitational Energy in the Gamma Metric", arXiv:gr-qc/0601141; O. Aydogdu and M. Salti, Prog. Theor. Phys. 115, 63 (2006); M. Salti and O. Aydogdu, "Energy in the Schwarzschild-de Sitter spacetime", arXiv:gr-qc/0512080; M. Salti, "On the energymomentum in closed universes", arXiv:gr-qc/0511095 M. Salti and O. Aydogdu, "Energy of a charged wormhole", arXiv:gr-qc/0511030, O. Aydogdu, M. Salti and M. Korunur, Acta Phys. Slov. 55, 537 (2005); M. Salti, Acta Phys. Slov. 55, 563 (2005); M. Salti, Nuovo Cim. 120B, 53 (2005); O. Aydogdu and M. Salti, Astrophys. Space Sci. 299, 227 (2005); M. Salti, Mod. Phys. Lett. A 20, 2175 (2005); M. Salti and A. Havare, Int. J. Mod. Phys. A 20, 2169 (2005); M. Salti and A. Havare, "Energy distribution in LTB space-time", arXiv:gr-qc/0502058; A. Havare, M. Salti and T. Yetkin, "On the energy-momentum densities of the cylindrically symmetric gravitational waves", arXiv:gr-qc/0502057 A. Havare, M. Korunur and M. Salti, Astrophys. Space Sci. 301, 43 (2006); O. Aydogdu, "Gravitational energy-momentum density in Bianchi type-II space-times", arXiv:gr-qc/0509047; O. Aydogdu, "Moller Energy-Momentum Prescription for a Locally Rotationally Symmetric Space-Time", arXiv:gr-qc/0601070; O. Aydogdu, "Energy Distribution of the Universe in the Bianchi type II Cosmological Models", arXiv:gr-qc/0602070 R.M. Gad, Astrophys. Space Sci. 293, 453 (2004); R.M. Gad, Astrophys. Space Sci. 295, 459 (2005); R.M. Gad, Astrophys. Space Sci. 295, 451 (2005); R.M. Gad, Mod. Phys. Lett. A 19, 1847 (2004); R.M. Gad, "Møller energy-momentum complex of a static axially symmetric vacuum space-time", gr-qc/0403039 R.M. Gad, "Energy and Momentum densities associated with solutions exhibiting directional type singularities", gr-qc/0404108; E.C. Vagenas, Int. J. Mod. Phys. A 18, 5781 (2003); E.C. Vagenas, Int. J. Mod. Phys. A 18, 5949 (2003); E.C. Vagenas, Mod. Phys. Lett. A 19, 213 (2004); O. Patashnick, Int. J. Mod. Phys. D 14, 1607 (2005); T. Gram- 
menos, Mod. Phys. Lett. A 20, 1741 (2005); I. C. Yang and C. H. Tsai, "The Evaluation of the Møller Energy Complex in Difference Coordinate Representations", arXiv:gr-qc/0503118 P. Halpern, "Black plane solutions and the Einstein energymomentum complex", arXiv:gr-qc/0505053.

[4] C.C. Chang, J.M. Nester and C.M. Chen, Phys. Rev. Lett. 83, 1897 (1999).

[5] G. Lessner, Gen. Rel. Grav. 28, 527 (1996).

[6] C. Møller, Ann. Phys. (N.Y.) 4, 347 (1958).

[7] R. Ruffini, "On the dyadosphere of black holes", arXiv:astro-ph/9811232.

[8] G. Preparata, R. Ruffini and S. S. Xue, Astron. Astrophys. 338, L87 (1998).

[9] S. L. Adler, Annals Phys. 67, 599 (1971).

[10] I. T. Drummond and S. J. Hathrell, Phys. Rev. D 22, 343 (1980).

[11] R. D. Daniels and G. M. Shore, Nucl. Phys. B 425, 634 (1994).

[12] V. A. De Lorenci, N. Figueiredo, H. H. Fliche and M. Novello, Astron. Astrophys. 369, 690 (2001).

[13] S. S. Xulu, "Energy distribution in the dyadosphere of a charged black hole", arXiv:gr-qc/0304081

[14] K.S. Virbhadra, Phys. Rev. D 41, 1086 (1990).

[15] K.S. Virbhadra, Phys. Rev. D 42, 2919 (1990).

[16] I. Radinschi, Mod. Phys. Lett. A 16, 673 (2001). 